\title{
Comparison between Virtualization and Cloud Computing
}

\author{
Babiker Mahialdean Salih ${ }^{1}$, Dr. Hala Aldaw Edreis ${ }^{2}$ \\ ${ }^{1,2}$ Faculty of Engineering, Neelain University, Khartoum-Sudan
}

\begin{abstract}
Virtualization is not the cloud. There are significant gaps between the capabilities of virtualization and the cloud, if you use virtualization technology to improve utilization of your computing resources, you may have heard that virtualization accomplishes the same goals as cloud computing. Not true. This paper analyzes the benefits of cloud computing, side-by-side with virtualization, and establishes why they are not interchangeable. If you want user empowerment, scalability, and relief from the burdens of maintaining hardware and software yourself, you want the cloud.
\end{abstract}

Keywords: Virtualization, cloud, Drawbacks, Deciding, Trace Analysis

\section{Introduction}

The virtualization concept is expanding into many aspects of information technology. Servers, switches, storage, networking, and clients are all on a virtualization roadmap, virtualization of business applications allows IT operations in companies of all sizes to reduce costs improve IT services and manage risk. The most dramatic cost savings are the result of reducing hardware, space and energy usage, as well as the productivity gains that also lead to cost savings. Service improvements include high availability of application workloads and rapid provisioning of new services to meet the dynamic needs of the business Virtualization can also mitigate risk to business operations. For example, when faults are detected or server load is too high, workloads can be moved out of harm's way ,Cloud Computing is a model for enabling ubiquitous, convenient, on-demand network access to a shared pool of configurable computing resources (e.g., networks, servers, storage, applications, and services) that can be rapidly provisioned and released with minimal management effort or service provider interaction.Virtualization is a way to make better use of your infrastructure, but it doesn't come close to the level of elasticity, empowerment, self-service, and automation of the cloud. Ultimately, with virtualization, you're still in the infrastructure business.

\section{Comparison between Virtualization and Cloud Computing}

\subsection{Benefits of Virtualization}

Virtualization emerged in the mainframe era as a practical solution to help IT departments make better use of their investments in enterprise-grade hardware, such as highperformance computers, servers, and storage. These machines could handle more workload than a typical application required, so the idea of running virtual machines, each performing discrete tasks, on a single piece of hardware, was appealing.

a) Client/server done right." In a way, virtualization is the last bastion of client/server technology. It has made that paradigm more efficient; think of it as "client/server done right." The virtualization universe ekes some additional value from enterprise-level architectures, concepts, and cost structures and makes running an IT estate operationally smoother. But fundamentally, it is still very much about deeply integrating applications with infrastructure, albeit with an additional layer of software that masks these distinctions.

b) Heavy duty. Virtualization nets benefits for enterprises with significant investments in traditional technologies such as large relational databases, virtual local area networks, multiyear byte servers, and storage area networks. The emphasis is on persistence and stability, and to a large degree, it was achieved.

c) New tools. Over time, tools were added to make administration of the virtual machines easier, such as templates, controllers for starting and stopping services, and centralized administration. Until recently, virtualization has not been very flexible, especially when it comes to enterprises' need to scale up or down quickly or to provision resources.

d) Privacy. For organizations with significant security requirements and a need for dedicated resources, virtual machines can replicate the required level of resource or device privacy and control that comes with hard-wired devices.

e) Customization. As virtualization is based on dedicated in-house resources, the potential for customization is extensive.

\subsection{Drawbacks of Virtualization}

Computing power to maximize efficiency of physical hardware is a concept that virtualization and cloud computing share, virtualization falls far short of the promise of cloud computing.

a) Virtualization does not offer self-service, choice, or empowerment. As an enterprise deployment, virtualization is complex and requires a high degree of centralized control, which means business users or departments can't simply "virtualized at will" and provision their own resources. A request still must be sent to IT, which adds time, when users could be spending that time innovating and experimenting.

b) Virtualization technology is not elastic.

A typical virtual machine environment is in place almost as long as a traditional client/server implementation and 


\section{International Journal of Science and Research (IJSR) \\ ISSN (Online): 2319-7064 \\ Index Copernicus Value (2013): 6.14 | Impact Factor (2015): 6.391}

is almost as difficult to deploy and retract. Starting and stopping services, and removing artifacts of virtual machines from hardware when they are no longer needed, is time-consuming and can result in costly errors. The license model doesn't support on-demand computing. Virtualization makes better use of computing power, but it still relies on a licensing model that mirrors enterprise software licensing, which can get very expensive, fast. To save money with virtualization, enterprises must first have expensive equipment inhouse, which is then logically subdivided. Licenses can't be turned on and off like a utility, which negates some of the value of the on-demand computing proposition of virtualization, you are still in the infrastructure business. Although you can get better bang for the buck out of your hardware with virtualization, you are still responsible for acquiring, managing, and replacing that hardware. IT staff still need to apportion the servers, administer change requests, and be responsible for the same patches, upgrades, and vendor roadmap concerns that traditional enterprise computing customers do.

c) Virtualization does not equal automation-at least not automatically.
It is possible to automate a virtualized infrastructure with certain additional tools, but they don't come out of the box.

d) Virtualization addresses only one pillar of IT service: Virtual machines are a good example of Infrastructureas-a-Service (IaaS), but they don't supply two other critical services that make enterprise computing run better: Platform-as-a-Service (PaaS) and Softwareas- aService (SaaS). Your enterprise is still on the hook for buying and managing hardware and software.

e) Virtualization doesn't boost security:

- While cloud providers have beefy security certifications for their data centers, it's a myth that virtual machines are inherently more secure than physical hardware. Securing 100 virtual machines requires the same amount of effort and diligence as securing 100 servers.

- Ultimately, virtualization was introduced to lower expenses and make life easier for IT, but not necessarily for everyone else. Because it is largely invisible or opaque to business users, it doesn't encourage self-service or pushing the boundaries of computing power to create new products and services.

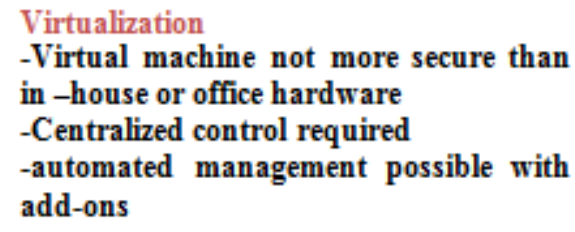

\subsection{The Advantage of the Cloud}

If your goal is to reduce technology cost, empower users, enable self-service, and get out of the IT infrastructure business, the answer lies in the cloud. Virtualization takes you only a small part of the way there.

a) The cloud automates management:

Fundamentally, the cloud's value is in automation. Virtualization was about moving from directly managing physical resources to managing virtual resources on top of physical resources. The step from virtual to cloud is a bigger and more fundamental transition. In the cloud, you can eliminate a high percentage of the IT tasks you used to have with on-premise equipment. Your equipment lives offsite, maintained by someone else. Through automated routines and application program interfaces, provisioning and managing resources is a snap. The cloud is largely self-managing and self-healing.

b) The cloud distributes control :

With virtualization's centralized control model, the organization still depends on one or two people sitting at a console. In the cloud, you can provide access to any number of developers and business users. IT managers can see, at a glance, exactly which applications are running where and how much computing power (therefore, how much money) they're consuming - and take action if that needs to change. Can you do that in your enterprise now c) The cloud scales :

Today, you might need 10 servers. Tomorrow, you might need 100. Because cloud providers have spent years building out data centers with highly scalable hardware, the cloud can accommodate wide swings in demand. Through easily accessible portals, you can switch services on and off, without having to submit a work ticket or wait for approval.

d) The cloud empowers:

The cloud empowers users to innovate, using massive amounts of commodity hardware, housed at secure data centers offsite. You don't have to wait for IT to locate and apportion computing power within your enterprise's finite resource base. If the marketing department wants to test a website concept (or five) with real market participants, they should be able to spin it up directly and immediately and switch it off as soon as the experiment is over. The faster you can access computing power when you need it, the faster you can innovate, without spending a lot of money.

e) The cloud supersedes virtualization

Conceptually, cloud computing and virtualization are similar. Both logically separate hardware, software, and user interface functions. Some cloud services, such as IaaS, use virtualization as a delivery mechanism. But the cloud continues where virtualization leaves off. With the cloud, you get infrastructure (IaaS), development platforms (PaaS), and applications (SaaS) delivered as a service, and the daily grind of maintaining those resources is no longer your problem. Virtualization is a 


\section{International Journal of Science and Research (IJSR) \\ ISSN (Online): 2319-7064 \\ Index Copernicus Value (2013): 6.14 | Impact Factor (2015): 6.391}

layer of software on top of your existing resources, which you must continue to maintain. The cloud removes the burden of maintaining those resources, and scales them infinitely, for you to use a service, when you need it. Don't let anyone tell you they're interchangeable.

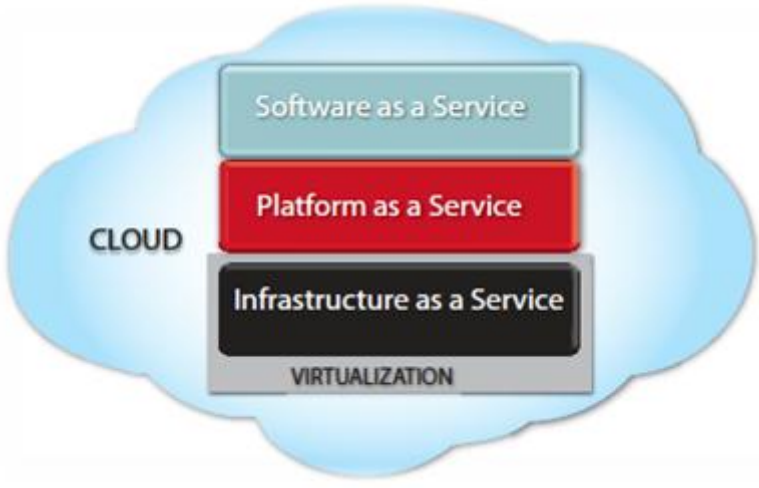

f) Private clouds can support virtualization

A managed private cloud provides dedicated infrastructure that can scale to your specific computing, bandwidth, and storage needs, fully supporting your virtual machines. Operating systems and application platforms are fully customizable. In a managed private cloud, you can get customized virtual hardware allocation and license renewal, round-the-clock data center support, the option to add resources when you need them, and service-level agreements. A managed private cloud can offer the best of both worlds.

\subsection{Deciding Between Cloud and Virtualization}

The Table below can help you If you're trying to decide what's right for your environment

\begin{tabular}{|l|c|r|}
\hline \multicolumn{1}{|c|}{ Need } & Virtualization & Cloud \\
\hline Increase Service Availability & $\checkmark$ & \\
\hline Consolidate hardware footprint & $\checkmark$ & \\
\hline $\begin{array}{l}\text { Move legacy applications onto } \\
\text { modern hardware }\end{array}$ & $\checkmark$ & \\
\hline $\begin{array}{l}\text { Increase scalability of existing } \\
\text { application }\end{array}$ & & $\checkmark$ \\
\hline $\begin{array}{l}\text { Expand and contract capacity on } \\
\text { demand }\end{array}$ & & $\checkmark$ \\
\hline Increase customer self-service & & $\checkmark$ \\
\hline Support dynamic workloads & & $\checkmark$ \\
\hline Support parallel workload & & $\checkmark$ \\
\hline
\end{tabular}

V.1 - Scalability : is very important for the company because it's mean the capability of a system, network, or process to handle a growing amount of work, or its potential to be enlarged in order to accommodate that growth.[1] For example, it can refer to the capability of a system to increase its total output under an increased load when resources (typically hardware) are added. An analogous meaning is implied when the word is used in an economic context, where scalability of a company implies that the underlying business model offers the potential for economic growth within the company.

V.2 - Support dynamic workloads: Cloud computing is a paradigm that harnesses massive resource capacity of data centers to support applications in a scalable, flexible, reliable and cost-effective manner. Despite its recent success and rapid adoption in the IT industry, recent literature has shown that effective workload management in cloud computing environments remains to be a difficult challenge. A key reason behind this difficulty is that resources and workloads in production environments are both heterogeneous and dynamic. In particular, large cloud data centers often consist of machines with heterogeneous capacities and performance characteristics. At the same time,

\subsection{For Example :Trace Analysis}

Workload traces collected from a production compute Cluster in Google over 29 days

- 12,000 machines

- 2,012,242 jobs

- 25,462,157 tasks

- Applications are represented by jobs

- User-facing jobs: e.g., 3-tier web applications

- Batch jobs: e.g., MapReduce jobs

- Each job consists of one or more tasks

- There are 12 priorities that are divided into three priority groups: gratis(0-1), other(2-8), production(9-11)

\section{Trace Analysis: Total Resource}

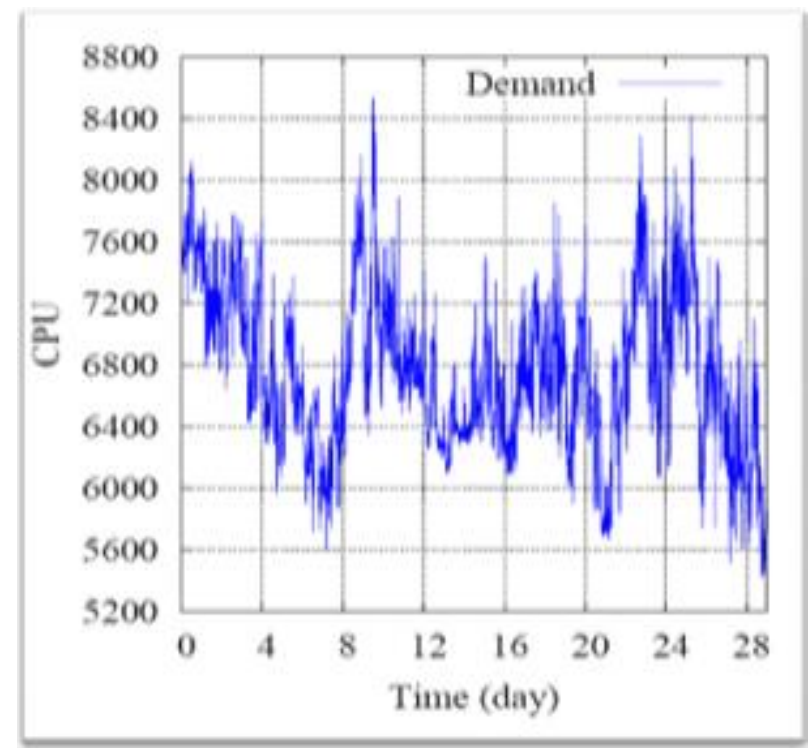

Figure: Total resource demand in Google's Cluster Data Set CPU Demand over 30 days

\section{Volume 5 Issue 6, June 2016 www.ijsr.net}




\section{International Journal of Science and Research (IJSR) \\ ISSN (Online): 2319-7064}

Index Copernicus Value (2013): 6.14 | Impact Factor (2015): 6.391

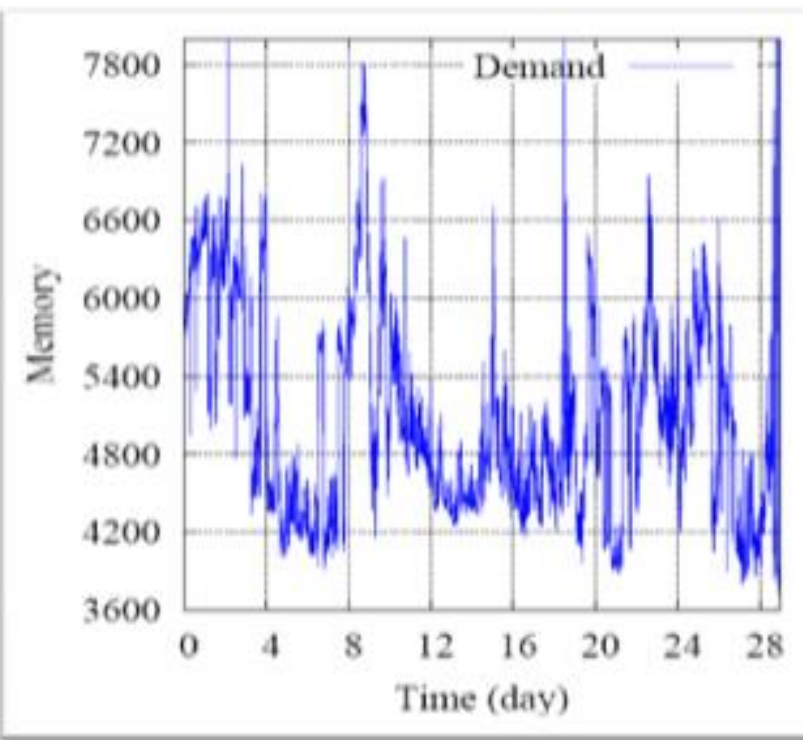

Figure: Total resource demand in Google's Cluster Data Set Memory Demand over 30 days

\section{Conclusion}

Virtualization is a layer of software that improves the management of the resources you already have. It conserves resources and makes some aspects of computing operationally smoother, but it doesn't relieve any of the responsibilities of IT. In fact, it adds one-managing virtualization itself. Nor does it provide self-service, elasticity, or automation for business users and developers.

Fundamentally, with the cloud, you're paying to use resources, located elsewhere, as a service. That means automation, user-friendly access, a full accounting of what's running where and how much it's costing you. With the cloud, you have a low-cost, robust, secure resource that expands when you need more of it and contracts when you need less. You're out of the infrastructure business and back where you belong - in the innovation business.

The cloud is as much a philosophy as a technology, and it's quite different from the philosophy that informs virtualization. The two should never be confused, but that doesn't mean they can't be combined. Managed private cloud services combine the flexibility of the cloud with the dedicated resources and customization capabilities of virtualization.

\section{References}

[1] Virtualization is not the Cloud - Contributed by Rackspace Engineers and Jacob Walcik. Jacob Walcik is a enterprise architect on the Rackspace Cloud Builders team. Having been at Rackspace a little over five years, Jacob enjoys the technical challenges, working with brilliant people and helping customers.

[2] http://csrc.nist.gov/publications/nistpubs/800145/SP800-145.pdf

[3] M. Haghighat, S. Zonouz, \& M. Abdel-Mottaleb (2015). CloudID: Trustworthy Cloud-based and CrossEnterprise Biometric Identification. Expert Systems with Applications, 42(21), 7905-7916.
[4] "What is Cloud Computing?". Amazon Web Services. 2013-03-19. Retrieved 2013-03-20.

[5] Baburajan, Rajani (2011-08-24). "The Rising Cloud Storage Market Opportunity Strengthens Vendors". It.tmcnet.com. Retrieved 2011-12-02.

[6] Oestreich, Ken, (2010-11-15). "Converged Infrastructure". CTO Forum. Thectoforum.com. Retrieved 2011-12-02.

[7] "Where's The Rub: Cloud Computing's Hidden Costs". 2014-02-27. Retrieved 2014-07-14.

[8] "Cloud Computing: Clash of the clouds". The Economist. 2009-10-15. Retrieved 2009-11-03.

[9] "Gartner Says Cloud Computing Will Be As Influential As E-business". Gartner. Retrieved 2010-08-22.

[10] Gruman, Galen (2008-04-07). "What cloud computing really means". InfoWorld. Retrieved 2009-06-02.

[11] "The economy is flat so why are financials Cloud vendors growing at more than 90 percent per annum?". FSN. March 5, 2013.

[12] "Realization of Interoperability \& Portability Among Open Clouds by Using Agent's Mobility \& Intelligence - TechRepublic". TechRepublic. Retrieved 2015-10-24.

[13] "Interoperability and Portability among Open Clouds Using FIPA Agent / 978-3-659-24863-4 / 9783659248634 / 3659248630". www.lappublishing.com. Retrieved 2015-10-24.

[14] Hassan, Qusay F.; Riad, laa M.; Hassan, Ahmed E. (2012). "Software reuse in the emerging cloud computing era". In Yang, Hongji; Liu, Xiaodong. Understanding Cloud Computing (PDF). Hershey, PA: Information Science Reference. pp. 204-227. doi:10.4018/978-1-4666-0897-9.ch009. ISBN 978-14666-0897-9. Retrieved 11 December 2014.

[15] Schmidt, Eric; Rosenberg, Jonathan (2014). How Google Works. Grand Central Publishing. p. 11. ISBN 978-1-4555-6059-2.

[16] "Internet History 1977".

[17] "National Science Foundation, "Diagram of CSNET," $1981 "$.

[18] Steven Levy (April 1994). "Bill and Andy's Excellent Adventure II". Wired.

[19] Antonio Regalado (31 October 2011). "Who Coined 'Cloud Computing'?". Technology Review (MIT). Retrieved 31 July 2013.

[20] "Announcing Amazon Elastic Compute Cloud (Amazon EC2) - beta". Amazon.com. 2006-08-24. Retrieved 2014-05-31.

[21] White, J.E. "Network Specifications for Remote Job Entry and Remote Job Output Retrieval at UCSB". tools.ietf.org. Retrieved 2016-03-21.

[22] "July, 1993 meeting report from the IP over ATM working group of the IETF". CH: Switch. Retrieved 2010-08-22.

[23] Corbató, Fernando J. "An Experimental Time-Sharing System". SJCC Proceedings. MIT. Retrieved 3 July 2012.

[24] Rochwerger, B.; Breitgand, D.; Levy, E.; Galis, A.; Nagin, K.; Llorente, I. M.; Montero, R.; Wolfsthal, Y.; Elmroth, E.; Caceres, J.; Ben-Yehuda, M.; Emmerich, W.; Galan, F. "The Reservoir model and architecture for open federated cloud computing". IBM Journal of Research and Development 53 (4): 4:1-4:11. doi:10.1147/JRD.2009.5429058. 


\section{International Journal of Science and Research (IJSR) \\ ISSN (Online): 2319-7064}

Index Copernicus Value (2013): 6.14 | Impact Factor (2015): 6.391

[25] Kyriazis, D; A Menychtas; G Kousiouris; K Oberle; T Voith; M Boniface; E Oliveros; T Cucinotta; S Berger (November 2010). "A Real-time Service Oriented Infrastructure". International Conference on Real-Time and Embedded Systems (RTES 2010) (Singapore).

[26] Dynamic Workload Management in HeterogeneousCloud Computing Environments Qi Zhang and Raouf Boutaba University of Waterloo IEEE/IFIP Network Operations and Management Symposium

Volume 5 Issue 6, June 2016 www.ijsr.net 